\title{
Fibre Bragg grating sensors in polymer optical fibres
}

David J Webb

Aston Institute of Photonic Technologies, Aston University, Birmingham, B4 7ET, UK

\begin{abstract}
This review paper summarises the current state of research into polymer optical fibre grating sensors. The properties of polymers are explored to identify situations where polymers offer potential advantages over more conventional silica fibre sensing technology. Photosensitivity is discussed and the sensitivities of polymer fibre gratings to strain, temperature and water are described. Finally, applications are reported which utilise the unique properties of polymer fibres.
\end{abstract}

\section{Introduction}

Optical fibre Bragg gratings (FBGs) take the form of a periodic (or quasi-periodic) modulation of the refractive index along the core of an optical fibre, which is usually single mode. The structure is normally produced by exploiting the intrinsic sensitivity of the core material to ultraviolet (UV) light and exposing the fibre to a periodic intensity pattern produced by interfering two beams of UV light. The FBG preferentially reflects light with a wavelength, $\lambda_{\mathrm{B}}$, determined by the Bragg condition [1]

$\lambda_{B}=2 n \Lambda$,

where $\mathrm{n}$ is the effective index of the guided mode and $\Lambda$ the spatial period of the modulation. For sensing applications, these devices are of interest because any strain or temperature applied to the fibre changes both the period and index of the grating leading to a shift in the reflected wavelength which can be determined by a spectroscopic measurement of some kind.

Over the last 25 years, the technology has become increasingly mature and is being commercially exploited in various fields that are each in some way able to exploit the technology's key features:

- Low fibre loss - enabling remote operation

- Immunity to electromagnetic interference - permitting use in electrically noisy environments

- Small size - allowing the sensors to be embedded in structures

- Multiplexing capability - with some schemes several hundred sensors can be addressed in a single fibre

The vast majority of the research on this technology - and all the commercial activity - has so far involved grating sensors inscribed in silica optical fibres. These developments are well described in books [1, 2] and review articles[3-6].

More recently, there has been growing interest in the development of the technology in polymer optical fibres (POFs). The motivation here lies with the rather different material properties of polymers compared to silica, which may provide advantages in certain applications. This paper seeks to contrast the behaviour of sensors in the two fibre types and summarise the current state of research into grating sensors in polymer optical fibres as well as the applications that have been suggested for them. Throughout this paper, when mentioning POF, we will by default be discussing 
fibres fabricated mainly from poly(methyl methacrylate), with other material being specifically highlighted. We should also note that whilst the focus of this paper is polymer optical fibre Bragg gratings (POFBGs), there have been reports of studies involving long period gratings[7, 8].

There are some review articles relevant to POFBG technology. Zubia and Arue [9] and Ziemann et al. [10] provide background information on the properties and manufacture of POF, while POF based sensors were reviewed by Peters in 2011 [11]. The latter article includes a description of some of the early development of POF based gratings.

\section{POF vs silica}

In this section we explore the different material properties of POF and silica fibre in order to identify situations in which POF may offer some advantage.

\subsection{Failure strain}

Pristine silica fibre typically has a failure strain of between 5 and $10 \%$ [12]. It is important to note though that it is challenging to obtain such values with grating sensors; great care must be taken to ensure there is no mechanical damage to the fibre surface during the inscription process [13], providing motivation for the production of FBGs on the draw tower during fibre manufacture [14]. Furthermore, repetitive loading can significantly decrease the failure strain. In the case of POF, failure strains of over $100 \%$ have been obtained [15], though this is critically dependent on the fibre production process. Annealing (holding the fibre at elevated temperate around 80 degrees for several hours) can be used to improve the tensile properties of the fibre [16]. The yield strain - the limit of quasi-elastic behaviour - is usually around 6\% [17].

\subsection{Elastic modulus}

The Young's modulus for silica is $73 \mathrm{GPa}[18]$, while for PMMA values of around $3.3 \mathrm{GPa}$ are typical [19]. It is important to note though that polymers are viscoelastic materials and the challenges this provides are discussed later. The very different values for the modulus renders a POF based sensor much more sensitive to axial force than a silica fibre of equal diameter. Perhaps more importantly, the difference is significant when FBGs are used to monitor structures that are themselves rather compliant; in this case a relatively stiff silica fibre attached to the structure can locally reinforce it and therefore report a much lower strain than would exist if the fibre were absent[20]. The lower Young's modulus of POF significantly reduces this effect.

\subsection{Water sensitivity}

PMMA displays an affinity for water, absorbing $2 \%$ at $23^{\circ} \mathrm{C}$ [19]. The absorbed water raises the refractive index of the fibre and causes swelling, both leading to an increase in the Bragg wavelength of any inscribed grating [21]. This property can be used to sense the amount of water in air [21], salt solution [22], and even fuel [23]. Of course, a sensitivity to water can be a serious disadvantage for some applications, e.g. for strain sensing. Fortunately there are polymers that are suitable for fibre manufacture that do not display significant water sensitivity [24]. 


\subsection{Chemical composition}

Polymers, being organic materials, can be modified using the vast range of tools from organic chemistry and if necessary this can be done at the preform stage before drawing, which occurs at a much lower temperature $\left(\sim 200^{\circ} \mathrm{C}\right)$ than for silica fibre. This is not an approach that has been much exploited as yet, but offers plenty of scope for providing optical amplification[25], enhancing nonlinearities[26] and functionalising the POF to selectively respond to different chemical or biochemical species[27].

\subsection{Fibre breakage}

A final potential advantage relates to the use of POF sensors for in-vivo medical sensing: in the advent of a fibre breakage, a polymer fibre will not produce a dangerous sharp.

\subsection{Challenges for POF based sensors}

Grating sensors based on POF have a number of issues which must be addressed if advantage is going to be taken of the positive features just described. The first concerns the fibre loss, which we typically measure in our laboratory to be around $1 \mathrm{~dB} / \mathrm{cm}$ at $1550 \mathrm{~nm}$ for single mode PMMA based POF. The loss falls off though as we move down in wavelength towards the visible region, reaching $0.2 \mathrm{~dB} / \mathrm{m}$ at $650 \mathrm{~nm}$ with large core fibre [28]. Single mode fibre currently tends to have a higher loss of a few $\mathrm{dB} / \mathrm{m}$ at this wavelength [29], probably due to the influence of scattering at the core cladding boundary, which increases in importance as the core size is reduced. A route to improved performance is the use of perfluorinated fibre, which can provide losses down to $10 \mathrm{~dB} / \mathrm{km}$ in the $1500 \mathrm{~nm}$ region[30] and in which the first gratings were recently reported.

Polymers are visco-elastic materials and this behaviour certainly complicates their use in strain sensors. Firstly, the yield strength and tensile strength have both been shown to increase with increasing strain rate [16]. Secondly, polymers display creep (where under the influence of a constant force the length gradually increases) and stress relaxation (a gradual reduction in the stress or tension when a sample is subjected to a constant strain). When POFBGs are used as strain sensors, this can lead to hysteresis in the sensor response to increasing and decreasing strain as shown in Figure 1. When the strain was reduced the readings diverged significantly at low strain values and in fact in this region the fibre was seen to be hanging loose between its two suspension points, implying there was no longer any tension. Following this experiment it took about 48 hours for the sensor to relax back to close to its original Bragg wavelength. 


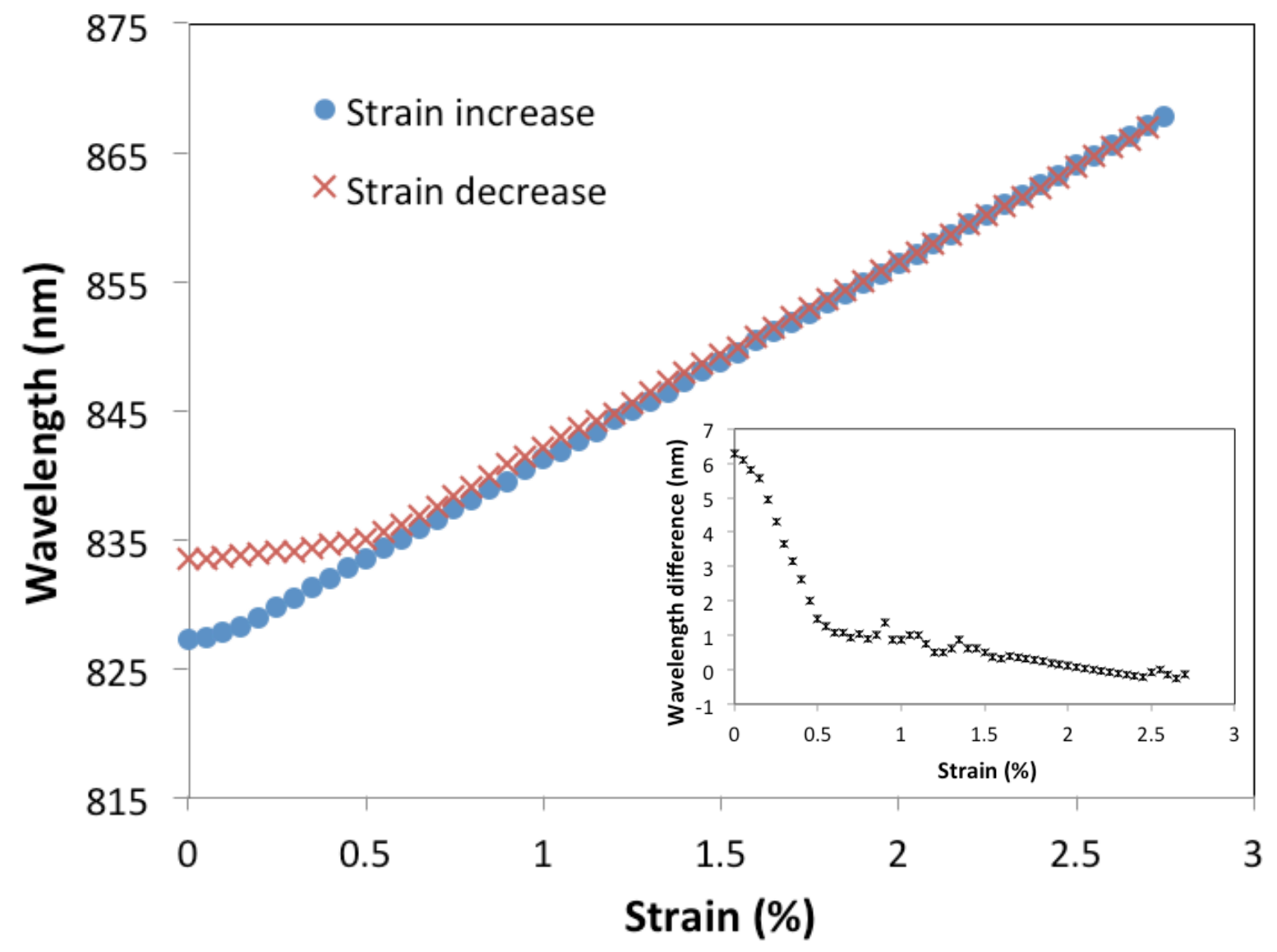

Figure 1 Bragg wavelength versus strain for a POFBG sensor in PMMA fibre. The sensor was strained by suspending it between two posts, one of which was mounted on a translation stage. The sensor was held at each strain point for 30 seconds[31]. Inset shows the wavelength difference between readings taken increasing and decreasing the strain.

This kind of behaviour is clearly undesirable in a strain sensor. Fortunately, in many applications the sensor is likely to be directly bonded to the substrate being monitored, or even embedded in the substrate. In this case, the substrate effectively forces the sensing fibre back to its original geometry. This is illustrated in Figure 2 where a similar experiment to that in Figure 1 was carried out but with the grating region of the fibre glued directly to a plastic beam that was then bent to induce the strain. The hysteresis has almost completely disappeared and what remains (as well as the small nonlinearity in the response that is visible) may be due to the visco-elastic properties of the plastic beam used, rather than the sensing fibre. 


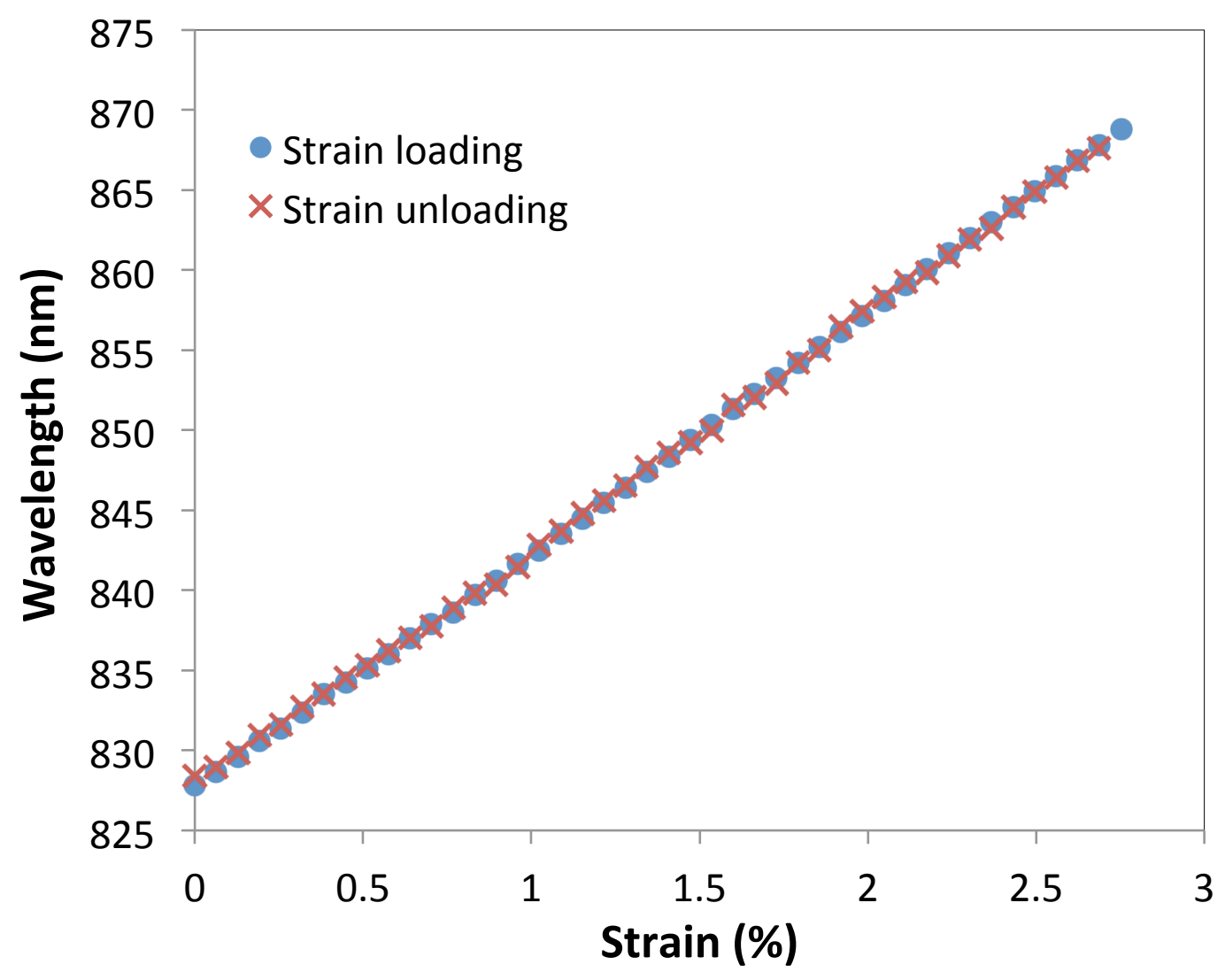

Figure 2 Strain characteristics of POFBG bonded to surface of beam[31].

The performance of POF based strain sensors can be affected in other ways. Annealing the fibre for several days at $95{ }^{\circ} \mathrm{C}$ tends to reduce the Young's modulus, the yield point and the tensile strength whilst increasing the failure strain [16]. Annealing the fibre and applying a pretension have also both been shown to reduce the amount of hysteresis exhibited by POF strain sensors[32].

The effects of annealing on the fibre arise as a consequence of the fibre drawing process, in which the tension used to draw the fibre from the oven results in an axial stress being present in the melt zone, which preferentially aligns the long polymer chains along the fibre axis. As a consequence, the drawn fibre is structurally anisotropic, to a degree that depends on the magnitude of the drawing stress. The fibre anisotropy reveals itself in several ways. Firstly, the fibre is birefringent when observed normal to its axis; in one set of experiments, the transverse birefringence of PMMA fibre was observed to increase from $1 \times 10^{-4}$ to $8 \times 10^{-4}$ as the drawing stress increased from $2 \mathrm{MPa}$ to 27 MPa [33]. Secondly, POFBGs display a positive wavelength with pressure that can be explained by the fibre being a transverse isotropic material[34]. Thirdly, thermal expansion along the fibre axis can be smaller than in directions normal to the axis[35]. Annealing the fibre allows the molecules to relax back towards a more isotropic configuration, reducing all these effects. For example, in one experiment with PMMA fibre, annealing at $100^{\circ} \mathrm{C}$ for 20 hours reduced the birefringence of fibre drawn under high stress (>20 MPa) by between 55 and $70 \%$ [30]. 


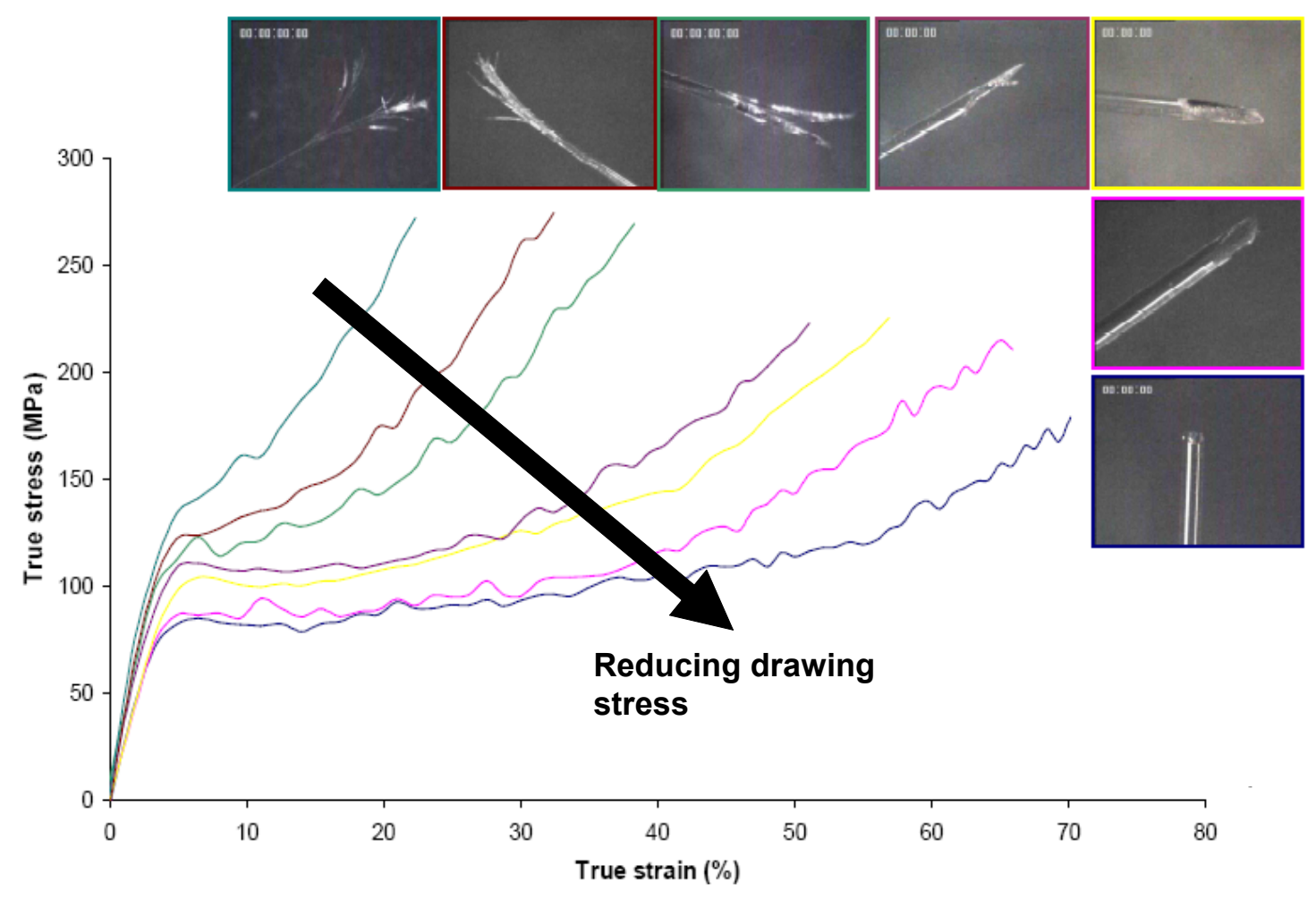

Figure 3 Effect of drawing stress on fibre properties[15].

Controlling the stress in the fibre during the drawing process has a significant effect on the stressstrain behaviour of the fibre, as shown in Figure 3[15]. Here it may be seen that as the drawing stress is reduced (achieved by increasing the oven temperature) the breaking strain increases and the Young's modulus decreases. Another feature is the changing morphology of the fibre end following failure, as shown in the inset photographs. Related to this is the observation in our laboratory that when fibre is drawn under high stress and later cut for connection purposes, the initially smooth fibre end can develop a frayed structure over time, ending up looking like the photographs towards the top left of Figure 3.

A final issue that should be mentioned is that POF has a much smaller usable temperature range than silica fibre, though the $110{ }^{\circ} \mathrm{C}$ upper limit reported for gratings in TOPAS fibre [36]should be sufficient for many applications.

\section{Photosensitivity and inscription}

The techniques used for inscription are essentially the same as those used for silica fibre, which are covered in several review papers cited in the introduction. The vast majority of groups use the phase mask approach, which minimises alignment and stability issues. There are two differences that sometimes need to be taken into account. Firstly, the high loss of POF in the $1550 \mathrm{~nm}$ region means that fibre lengths are typically restricted to around $5 \mathrm{~cm}$ and this may require the construction of a special fibre holding jig for the inscription set-up. Secondly, because the compliant nature of POF, if it is to be suspended horizontally between two supports, as is usual in silica fibre inscription set-ups, it is necessary to tension the fibre, leading to an inscription strain of several tens of me. This means that following inscription the Bragg wavelength will relax to a lower wavelength than that expected 
from the phase mask period. To overcome this problem, a vertical recording geometry can be used, with the fibre supported by horizontal v-grooves[37].

At the time of writing, the photosensitivity that permits the inscription of POFBGs is not fully understood, even if we restrict the discussion to PMMA illuminated by continuous wave UV light. Research on this topic dates back to the early 1970s when work was done on bulk PMMA at Bell Labs. Following illumination with $325 \mathrm{~nm}$ light from a HeCd laser, Tomlinson et al. discovered an increase in the refractive index of the polymer[38]. Index changes of over $10^{-3}$ were obtained, accompanied by a density increase of $0.8 \%$ and the group eventually attributed this process to the UV photo-polymerisation of unreacted methyl methacrylate monomers within the material[39], the role of the monomers later being confirmed by Marotz[40]. When relating this early work to current research on POFBGs it is important to keep in mind that whereas gratings in PMMA fibres typically form during UV illumination in a few tens of minutes, the index changes produced by the Bell Labs group developed over a period of a few hundred hours after illumination[41]. Furthermore, it was found to be necessary to polymerise the PMMA from monomer that had first been oxidised[38].

In 1984 Kopietz et al. studied the formation of gratings in PMMA induced by a mercury lamp[42]. They supported the role of photo-polymerisation in the inscription process, but also incorporated an initial generation of monomers to explain a decrease in refractive index that occurred prior to an increase of as much as 0.01 . A potential mechanism for the initial generation of monomers is provided by work from Mitsuoka et al., who investigated the photo-degradation of PMMA produced by a range of wavelengths around $300 \mathrm{~nm}[43]$. They noted that main chain scission could produce monomer and radical groups. Further evidence for what appears to be a competitive process between photo-degradation and photo-polymerisation is provided by recent work by Sáez-Rodríguez et al. who studied grating inscription in PMMA based mPOF[44]. They discovered a strong correlation between the strain applied to the fibre during inscription and the final grating reflectivity. They suggested this was strong evidence for the role of photo-degradation as the application of stress is known to significantly enhance photo-degradation[45].

There are other reports of grating inscription in the literature which complicate this picture, indicating perhaps that - as with silica FBG inscription - there are several possible mechanisms for photosensitivity, depending on the characteristics of the light source used as well as on the precise composition of the PMMA sample (molecular weight distribution, amount of unreacted monomer, degree of branching, presence of initiators etc.). Wochnowski used mass spectrometry to study the ablated products resulting from exposure of PMMA to UV light with wavelengths from $193 \mathrm{~nm}$ to $308 \mathrm{~nm}$ and SPS and FTIR spectroscopy to investigate the irradiated samples themselves[46]. They were able to detect side-chain scission at shorter wavelengths but surprisingly did not detect any polymer modification at $308 \mathrm{~nm}$. Working with fibres composed of PMMA doped with benzyl methacrylate, the group of G.D. Peng at the University of New South Wales, Australia, reported studies of grating inscription using a pulsed laser at $325 \mathrm{~nm}$. With an average intensity of 60 $\mathrm{mW} / \mathrm{cm}^{2}$ they observed two regimes: an initial linear increase in index modulation with time up to 60 minutes, followed by a much more rapid increase in modulation[47]. The first regime was characterised by complementary reflection and transmission spectra indicative of low loss, index gratings, while in the second regime losses occurred at wavelengths below the Bragg wavelength and damage was visible in the fibre. A blue wavelength shift of the Bragg peak was visible during inscription, suggesting a decrease in index was taking place. In a second paper, Peng's group 
observed radically different behaviour at a lower intensity of $45 \mathrm{~mW} / \mathrm{cm}^{2}[48]$. They reported an increase in reflectivity for 28 minutes after which the reflectivity remained roughly constant up to 48 minutes. The reflectivity then decreased until by 88 minutes there was virtually no reflected signal visible. The UV laser was then blocked and the reflectivity increased over about 8 hours, after which it remained constant.

Recording times for POFBGs in PMMA fibre are typically 10s of minutes, though careful adjustment of the inscription set-up can reduce this to less than 10 minutes[49]. Several groups have explored the use of dopants to improve the photosensitivity. As early as 1971 Laming[50] added pbenzoquinone to PMMA leading to photosensitivity at $488 \mathrm{~nm}$; the grating was then fixed by exposure to UV, with an index decrease of $1 \times 10^{-3}$ being obtained. Peng's group at the University of New South Wales, Australia, have experimented with a number of dopants; for example fluorescein was used to provide sensitivity to light at 488 and $524 \mathrm{~nm}$ [51]. Yu et al. have utilised trans-4stilbenemethanol, which exhibits UV-induced photo-isomerisation[52]. The same group reported the inscription of long period gratings (LPGs) with an index modulation as high as 0.02 using a mercury lamp and fibre doped with methyl vinyl ketone and benzyl methacrylate. Trans-4-stilbenemethanol has been used in the production of an MPOF targeted specifically at LPG fabrication; the dopant was added to the external part of the solid cladding of the fibre[53].

A $10 \%$ concentration of benzyl dimethyl ketal (BDK), a photo-initiator, was used by Peng et al.[54] to enhance UV induced photo-polymerisation leading to a positive index change of $4.5 \times 10^{-5}$, though this index change was nearly two orders of magnitude below that obtained with similarly doped PMMA films[55]. BDK was also used to dope the core of a PMMA based mPOF leading to an index change of $3.2 \times 10^{-4}$ and allowing a $23 \mathrm{~dB}$ grating to be written in 13 minutes[56].

The discussion above is limited to UV grating inscription in PMMA based fibre where most of the research on photosensitivity - and its improvement - has taken place. PMMA is not the only polymer that is suitable for fibre manufacture, nor the only one that exhibits UV photosensitivity. A very significant polymer currently being studied is TOPAS, a cyclic olefin copolymer[57][57][57][56][55][54]. This material has good optical properties, is suitable for fibre drawing and exhibits sensitivity to UV light at $325 \mathrm{~nm}$ [24]. Importantly, unlike the case with PMMA based fibres, POFBGs inscribed in TOPAS fibre are not significantly affected by the presence of water in the environment surrounding the fibre[58]. A recent development that is of great potential benefit is the production of POFBGs in perfluorinated fibre[59], in which the losses are significantly less than in PMMA. There do not appear to be any studies of the photosensitivity mechanism in TOPAS or perfluorinated PMMA fibre. Finally, it should be noted that "normal" photosensitivity is not required when inscription is carried out using high power, ultra-short (femtosecond) pulse lasers[60]. Inscription by such short pulse lasers may ultimately provide a path to low cost grating fabrication during the fibre drawing[14].

\section{Measurand sensitivity}

Silica FBGs are directly sensitive to strain induced by an axial stress, pressure induced by isotropic stress, temperature and axial magnetic field (though we will from now on ignore the last, as the effect is quite small[61]). POFBGS are sensitive to the same range of parameters and in addition, 
some polymers can respond to the presence of water. This is true of PMMA, which forms the basis of most POF.

\subsection{Strain}

There is some variation in the reported strain sensitivity of POFBGs. For example, for gratings in the $1550 \mathrm{~nm}$ region, values in the literature range from $1.15 \mathrm{pm} / \mu \varepsilon[62]$ up to $1.5 \mathrm{pm} / \mu \varepsilon[63]$, though most measurements seem to be in the range 1.3-1.4 pm/ $\mu \varepsilon[32]$. In comparison, the strain sensitivity of silica fibre FBGs in this region is about $1.2 \mathrm{pm} / \mu \varepsilon[64]$. With POF it is straightforward to obtain large strain tuning ranges; for example, $73 \mathrm{~nm}$ was achieved as early as the year 2000[63].

The much lower Young's modulus of POF compared to silica fibre means that POFBGs are much more sensitive to stress in the fibre and by using a POF etched down to 30 microns Rajan et al. achieved a force sensitivity of $643 \mathrm{~nm} / \mathrm{N}$ [65], with the added bonus that the inscription time of the grating was significantly smaller than for pristine fibre[66] (Hu et al. have noted that even a slight amount of etching can have a significant effect on fibre inscription time[67]). Finally we note that single mode POFs fabricated in research labs often have somewhat variable fibre parameters, with the diameter fluctuating along the fibre length and the core sometimes being off centre. This latter feature has been used to advantage as it renders the fibre intrinsically sensitive to bending, which induces a strain in the off-centre core[62].

Straining the fibre during the inscription process can be used to record gratings at different wavelengths using a single phase mask; in one study a useful strain range of $0.9 \%$ was obtained allowing gratings to be produced over a $12 \mathrm{~nm}$ range[68].

\subsection{Temperature}

Whilst the literature shows broad agreement over the strain sensitivities of POFBGs, there is a much greater range of reported values when it comes to temperature. In part this is because in the early days of the technology, many measurements were made in the open laboratory environment where the humidity was not controlled leading to cross sensitivity issues. In such experiments, sensitivities as high as $-360 \mathrm{pm} /{ }^{\circ} \mathrm{C}$ have been reported [69].

Over a sufficiently small temperature range to ensure a linear response - and in the absence of cross-sensitivity issues - the shift in the Bragg wavelength of an FBG is given by [56]

$\Delta \lambda_{B}=\lambda_{B}(\alpha+\xi) \Delta T$

where $\Delta \lambda_{B}$ is the Bragg wavelength shift, $\Delta T$ is the temperature change, $\lambda_{B}$ is the Bragg wavelength, $\alpha$ is the thermal expansion coefficient and $\xi$ is the thermo-optic coefficient. In the case of silica fibre, both coefficients are positive, ensuring a positive wavelength shift with temperature. For polymers, $\xi$ is negative so that the sign of the wavelength shift depends on whether refractive index change dominates over thermal expansion. Normally this is the case; in fact there is only one report of a (small) positive thermal sensitivity for a POFBG [70]. The sensitivity depends on the humidity environment of the fibre, with Harbach [21] reporting values ranging from $-10 \pm 0.5 \mathrm{pm} /{ }^{\circ} \mathrm{C}$ under dry conditions to $-36 \pm 2 \mathrm{pm} /{ }^{\circ} \mathrm{C}$ in water for a grating of nominal wavelength $1545 \mathrm{~nm}$. The precise value is also fibre dependent, for example Zhang et al. [71] have reported a sensitivity of $55 \mathrm{pm} /{ }^{\circ} \mathrm{C}$ at a constant $50 \%$ relative humidity, also for a grating in the $1550 \mathrm{~nm}$ region. Gratings fabricated in 
TOPAS based fibre, which is water insensitive show a similar thermal sensitivity, with $-37 \mathrm{pm} /{ }^{\circ} \mathrm{C}$ being reported for a grating recorded at $1568 \mathrm{~nm}$ [24].

Annealing of the fibre, either pre- or post-inscription, has a significant effect on the thermal sensitivity. In one experiment a grating inscribed in pristine fibre exhibited a reversible temperature response from room temperature only up about $55^{\circ} \mathrm{C}$ [72]. Beyond this temperature there was a rapid and permanent reduction in the Bragg wavelength associated with a reduction in the fibre length. By annealing the fibre, a repeatable working range up to over $80^{\circ} \mathrm{C}$ was obtained, though by this stage the room temperature Bragg wavelength had reduced by almost $20 \mathrm{~nm}$ from that before annealing. This annealing process has been used to tune the Bragg wavelength, enabling a wavelength multiplexed sensing system with three gratings to be fabricated using just one constant period phase mask [73].

\subsection{Water}
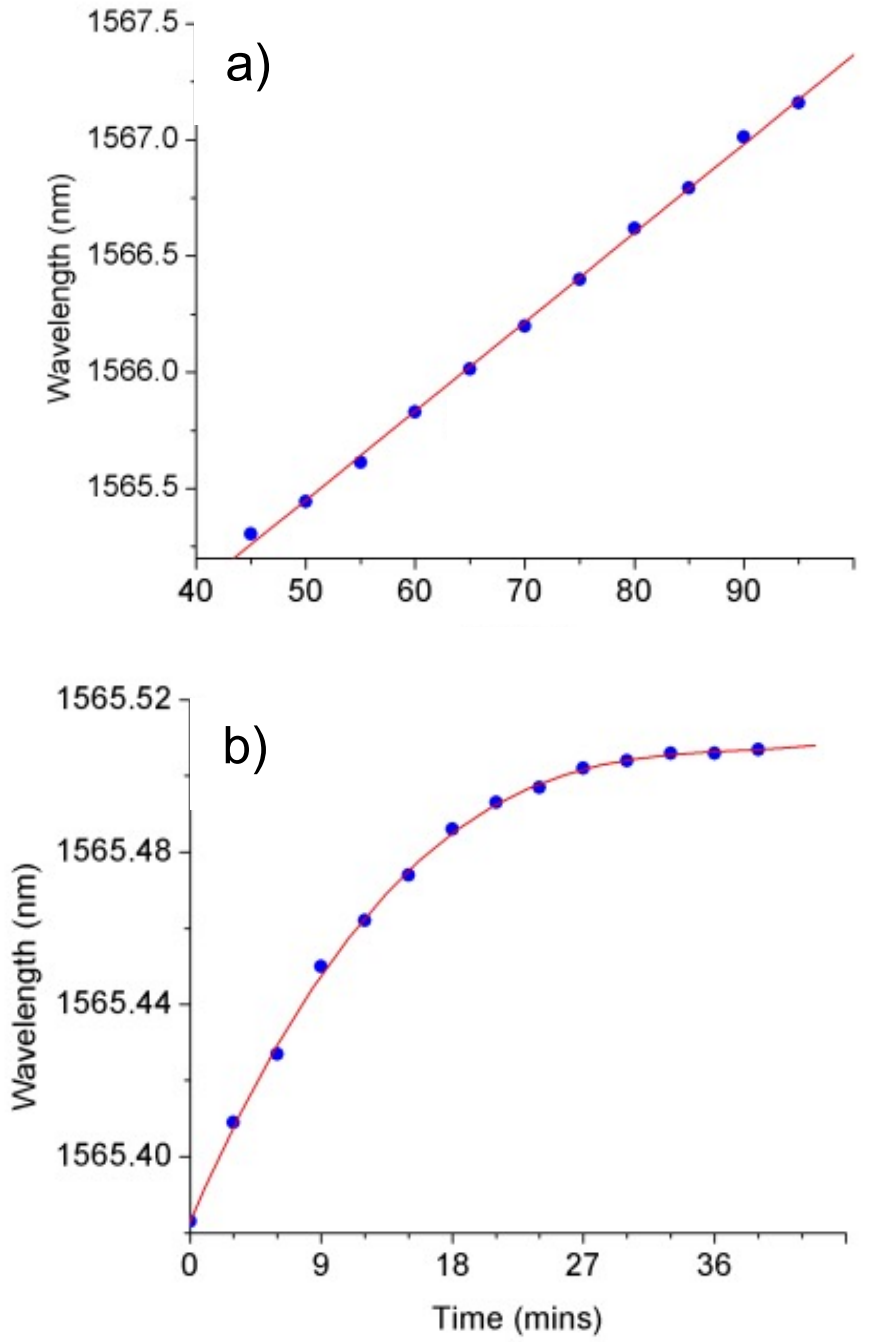

Figure 4 Humidity response at $22{ }^{\circ} \mathrm{C}$ of a POFBG recorded in PMMA based fibre. a) Wavelength shift. b) Response time. 
As previously mentioned, PMMA has an affinity for water, the absorption of which causes the fibre to swell and the index to rise, both leading to a positive shift in the Bragg wavelength [21]. This renders PMMA based POFBGs sensitive to humidity as shown in Figure 4, but POFBGs are also sensitive to aqueous solution concentration [22] and even water dissolved in aviation fuel [23].

The dependence of the Bragg wavelength on humidity is linked to that on temperature. For the Bragg wavelength shift arising as a result of a change in humidity $\Delta \mathrm{H}$ and a change in temperature $\Delta T$ we can write [74]

$\Delta \lambda_{B}=\lambda_{B}(\eta+\beta) \Delta H+\lambda_{B}(\xi+\alpha) \Delta T$

where $\eta$ is the normalized dependence of refractive index on humidity and $\beta$ the swelling coefficient related to humidity induced volumetric change $(\% \mathrm{RH})^{-1}$ (the other coefficients having been defined in equation 2). Complications arise because $\eta$ and $\beta$ are not constants. The former decreases approximately linearly with increasing temperature while the latter is independent of temperature, but only constant in the region $40-100 \% \mathrm{RH}$, decreasing below that range. Adding to this complexity is the fact that because the humidity response is partly due to water induced fibre expansion, it is also affected by any strain that is applied to the fibre [74].

As shown in Figure 4, the nominal response time of POFBGs to humidity tends to be a few tens of minutes. This does vary significantly from fibre to fibre, which is probably related to the differing molecular weight distributions of the fibres. Significant improvements to the response time have been achieved by etching down the fibre using acetone. Zhang et al. were able to improve the response time of one fibre from 31 to 12 minutes by reducing the diameter from 190 to 135 microns [75]. The authors noted a correlation between the rate at which the fibre was etched by the acetone and the response time to humidity and suggested that both were enhanced by a less tightly connected molecular structure. More recently Rajan et al. [76] etched a POFBG down to a diameter of just 25 microns, leading to a response time of just a few seconds.

It is important to note that the response of a POFBG to water in the surrounding environment is not directly related to the total amount of water present but rather to the water activity [77]. The water activity is defined as the fraction of the maximum water content in the surrounding medium, so the water activity is equal to 1 if the surrounding medium is fully saturated with water. If the surrounding medium is air, then the water activity is equal to the relative humidity, with $100 \%$ relative humidity corresponding to a few percent of water by mass at room temperature. If the surrounding medium happens to be aviation fuel then a water activity of 1 at room temperature corresponds to a few tens of parts per million. Both situations would lead to similar shifts in Bragg wavelength compared to a completely dry environment and for this reason POFBGs can provide a very sensitive measurement of water content in media that dissolve very little water. For example, with a POFBG operating in the $1550 \mathrm{~nm}$ region in air, a change in relative humidity of $50 \%$ results in a shift in Bragg wavelength of around $2 \mathrm{~nm}$ at room temperature [74]. A 50\% change in the dissolved water content of aviation fuel produces the same wavelength shift [78]. 


\section{Applications}

\subsection{Practical concerns}

In this section we focus on applications proposed for POFBGs, where the particular properties of polymers offer some advantage over the use of the better-established silica technology, but first we will discuss some of the practicalities involved in POFBG usage. Some things are on the face of it trivial and yet turn out to be important in handling POF. For example, silica fibre is either in one piece and functional or it is broken, whereas it is possible to snag a piece of single mode POF so that it remains unbroken but may have a sharp bend induced in it that prevents light guidance.

At the moment it is almost always necessary to connect POFBGs to a silica fibre lead, either because of the relatively high fibre losses of POF or in order to connect to a single mode coupler or a pigtailed source, which are only available with silica fibre. There is not yet a POF equivalent of the silica fibre splicer; instead it is necessary to either glue the fibres together[79] or connectorise the fibre[29, 80]. As previously noted, single mode POF samples made in research labs sometimes do not have a concentric core, making the glued connection the only option in this case. Finally, it should be noted that POF cannot be cleaved like silica fibre; instead it must be cut and studies have investigated the optimum approach for this, involving control of the fibre and blade temperature[81, 82].

\subsection{Tuneable filter}

The first application suggested for POFBGs made use of the wide strain tuning range that can easily be achieved with POF. A grating in PMMA based fibre was used as a mirror in a fibre laser system, where a tuning range of more than $35 \mathrm{~nm}$ was obtained in the $C$ band with an applied strain of just under 2.5\%[83]. The enhanced temperature sensitivity of POFBGs was also later exploited to produce a thermally tuneable filter[84]. A POFBG was coated at room temperature with a Pd/Cu film to permit electrical heating, with a sensitivity of $-13 \mathrm{pm} / \mathrm{mW}$ and a response time of 0.6 seconds being obtained for electrical powers up to 160. It has also been shown that the high absorption of POF in the $1550 \mathrm{~nm}$ region can permit optically induced temperature tuning of gratings[85].

\subsection{Compliant structures}

As mentioned earlier, when FBGs are used to monitor structures composed of low elastic modulus, POF sensors provide a truer representation of the strain in the material silica sensors, where the effect of the stiff fibre is to locally reinforce the structure. The first of such an application came from a project developing sensors for monitoring historic comparison was carried out on a representative textile sample, where POFBGs and silica glued to the sample using two types of glue: Araldite epoxy resin and DMC2 a compliant 


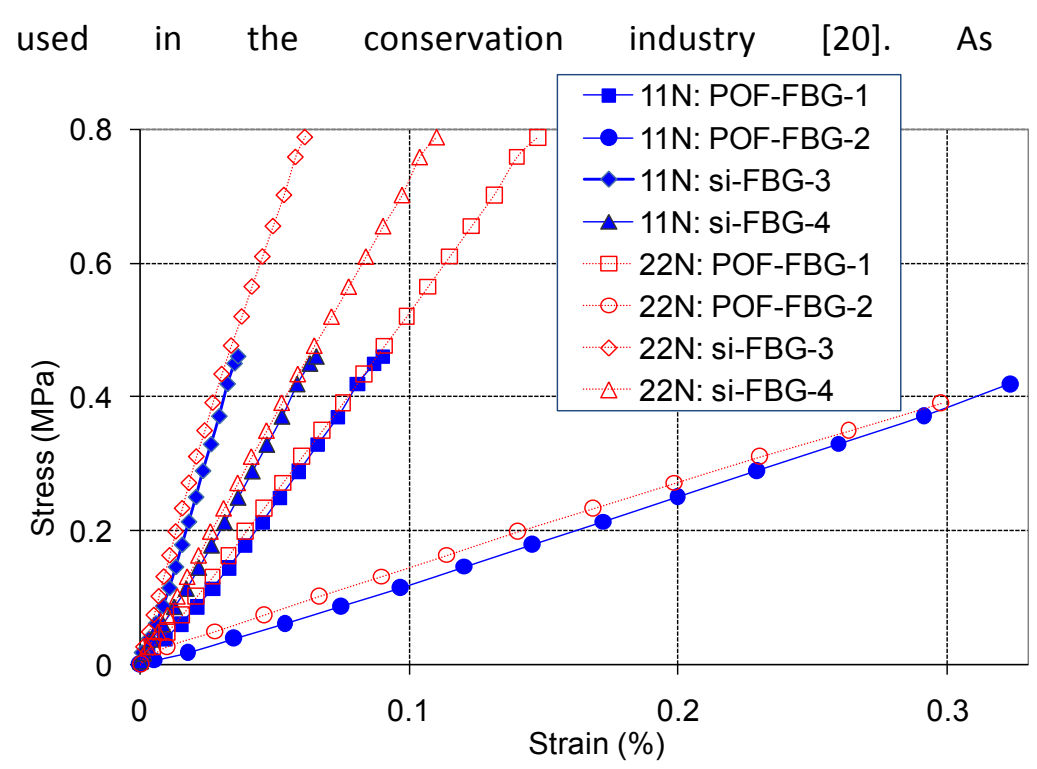

Figure 5, for a given stress, by far the highest strain is recovered by POF-FBG-2, a PMMA based sensor glued using DMC2.

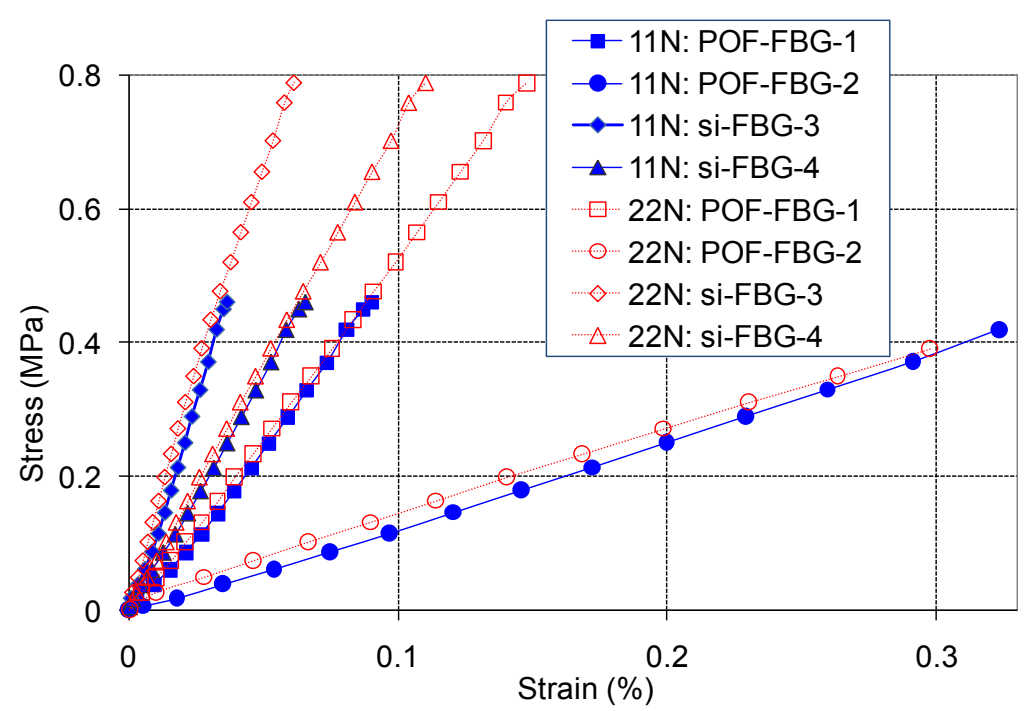

Figure 5 Stress strain curves for silica and POF gratings glued to a textile sample and subject to loads up to $11 \mathrm{~N}$ and $22 \mathrm{~N}[20]$. 
A further graphic example of the ability of POFBGs to monitor compliant structures was brought out in an experiment in which silica and POF Bragg gratings were embedded in thin sheets of poly dimethyl siloxane (PDMS) [86]. With the sheets lying on a flat horizontal surface a small weight was translated along the sheets directly above the fibre, with the response being shown in Figure 6. It may be seen that the POFBG experiences approximately 10 times the wavelength shift of the silica FBG, despite the intrinsic strain sensitivities of the fibre being comparable.

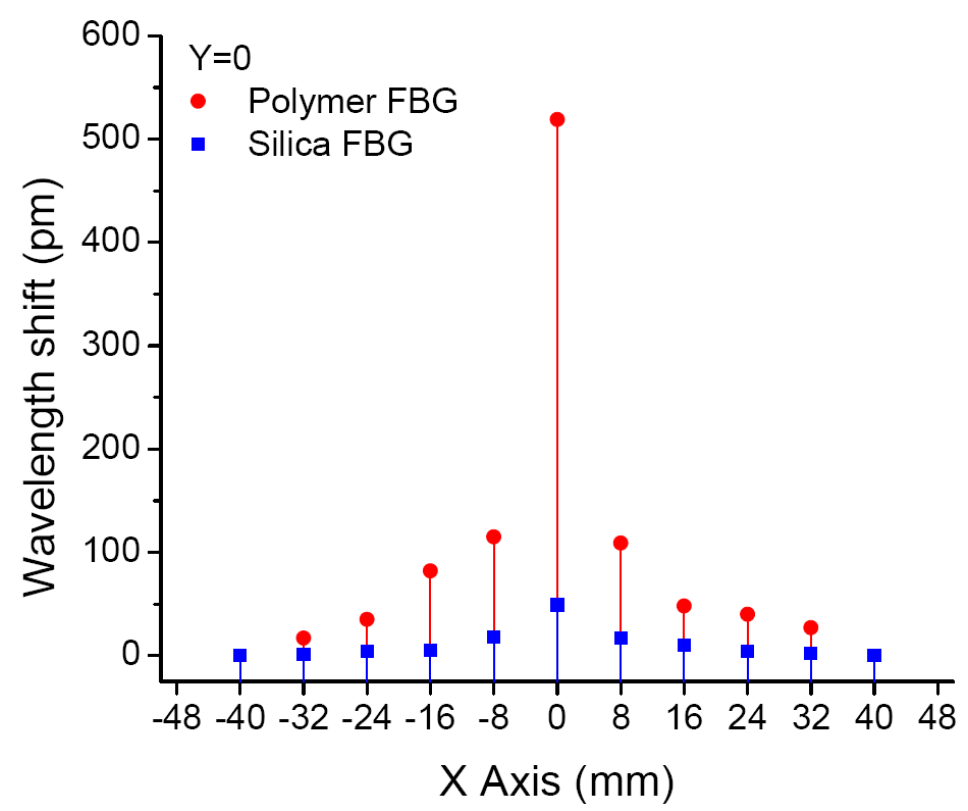

Figure 6 Comparison of the response to a weight translated over FBGs recorded in silica and POF and embedded in a PDMS sheet[86].

It has been shown that POFBGs can survive being embedded into composite materials. In one study, measurements of both the Bragg wavelength and the spectral width of the grating permitted discrimination between the temperature and the thermal expansion of the composite structure[87].

\subsection{Dynamic strain sensing}

The two previous examples concern the response of POFBGs to quasi-static strain. The low elastic modulus of POF suggests they may have advantages for use in dynamic applications, e.g. as acoustic sensors or accelerometers, however the viscoelastic nature of POF means that some caution must be exercised. Stefani et al studied both PMMA and TOPAS fibres, concluding that for dynamic strains up to $0.28 \%$ the Young's modulus was constant up to a frequency of $100 \mathrm{~Hz}$ - the limit of the measurement equipment - with very little sign of viscoelasticity [88]. The authors demonstrated an accelerometer with a flat frequency response to over $1 \mathrm{kHz}$, capable of sensing up to $15 \mathrm{~g}$ and with a sensitivity 4 times that of an equivalent silica FBG[89].

Gallego and Lamela have explored the potential for POF based sensors to detect ultrasound for medical applications, finding a POF based interferometer offered an intrinsic phase sensitivity about 15 times greater than an equivalent silica one[90]. Marques et al. investigated the use of acoustic waves launched onto a fibre to modify the spectral profile of a grating filter inscribed in the fibre. 
They found in both simulation and experiment that there was a much higher strain level in the POF compared to an equivalent silica system[91].

\subsection{Water}

As mentioned earlier, POFBGs recorded in PMMA based fibre respond to the water activity of the surrounding medium, displaying for example a strong dependency on the relative humidity of air. This property makes them sensitive to the concentration of any aqueous solution surrounding the fibre. Essentially an equilibrium is set up, balancing on the one hand the water affinity of the polymer and on the other the osmotic pressure of the surrounding solution, which seeks to draw water from the fibre. The dependence of the Bragg wavelength on the concentration of sugar and salt[22] solutions have both been demonstrated. An example of the latter is shown in Figure 7, where it may be seen firstly that significant changes in Bragg wavelength occur with varying concentration, and secondly that the process is reversible, with any small differences being consistent with the temperature stability of the laboratory.

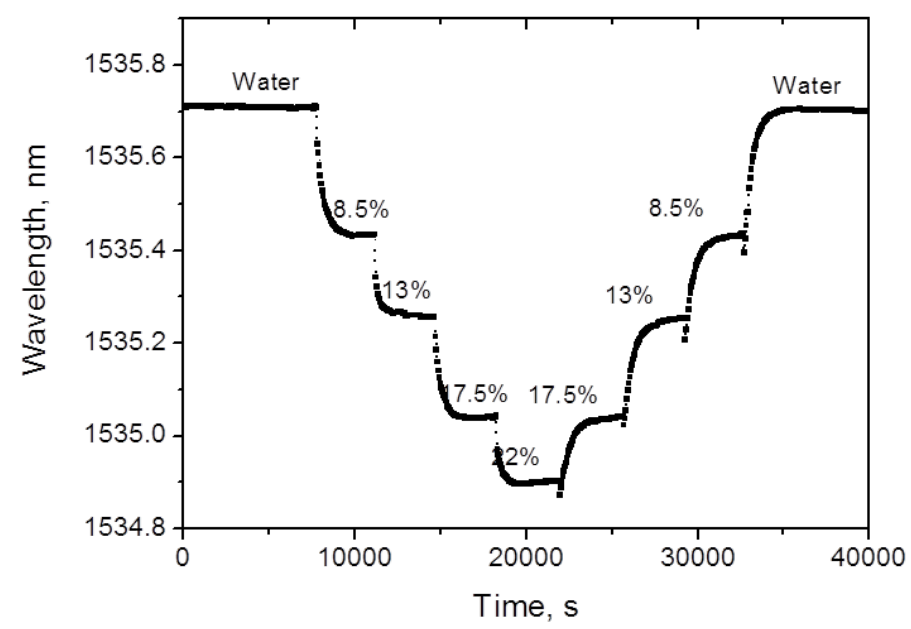

Figure 7 Bragg wavelength versus concentration of saline solution for a POFBG inscribed in PMMA based fibre[22].

The aviation industry places great emphasis on the minimisation of the amount of water in aircraft fuel tanks, since free water acts as an incubator for anaerobic microbial contamination and when frozen can in extreme circumstances block the fuel system. Water exists in a fuel system either dissolved in the fuel or else as free water lying at the bottom of the fuel tank or as an emulsion suspended within the fuel. Water can enter the fuel system in solution but as the temperature falls, the fuel can become saturated with water, after which the water condenses out as free water. The saturated water content is very low in absolute terms, e.g. about $70 \mathrm{ppm}$ at room temperature[92], but as this represents a water activity of 1 , it nevertheless results in a significant Bragg wavelength shift. Figure 8 shows the measured dependence of Bragg wavelength shift versus water content for a grating with a nominal wavelength of $1532 \mathrm{~nm}$. It may be seen that the sensor benefits from a linear response. 


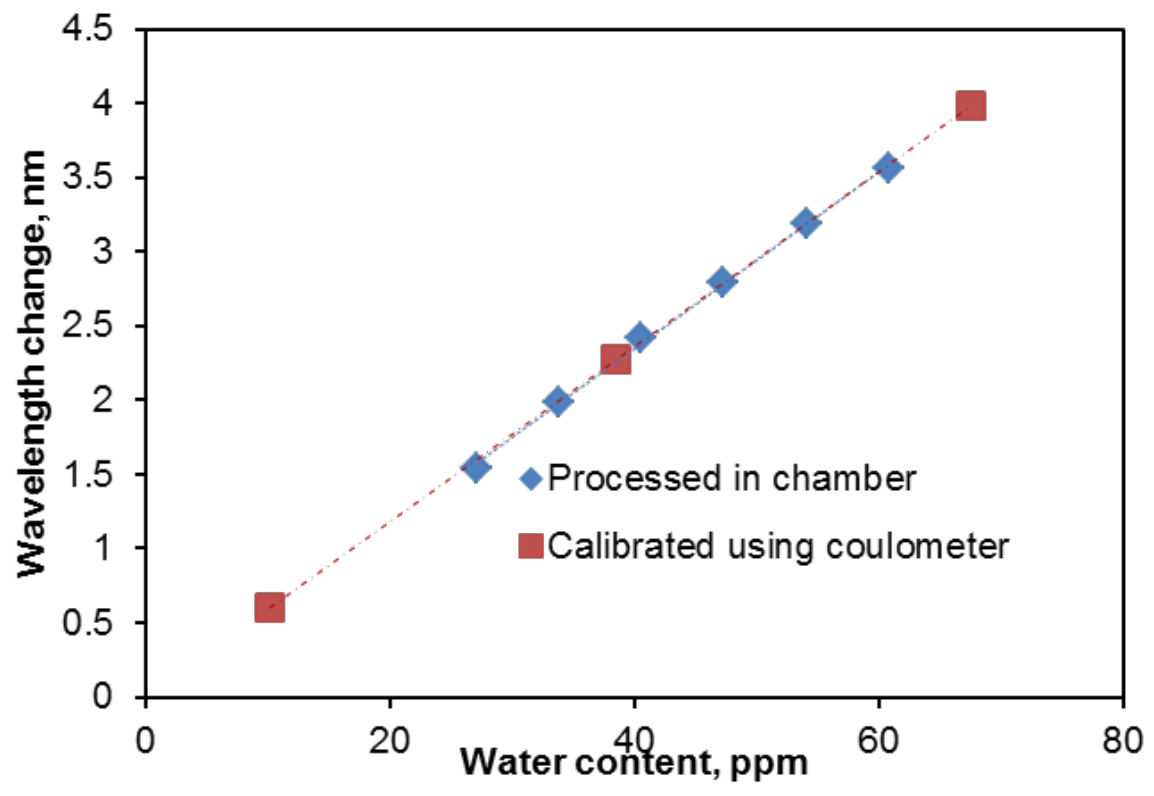

Figure 8 Bragg wavelength shift vs water content in Jet-A1 for a grating with a nominal wavelength of $1532 \mathrm{~nm}$.

The fact that PMMA POFBGs are sensitive to humidity while silica FBGs are not,, coupled with the different signs of their temperature sensitivities has been used to good effect in a combined humidity and temperature sensor, in which a silica FBG and a POFBG were juxtaposed either side of a glued connection between the two fibres. Measuring the wavelength shifts from both gratings allows the temperature and humidity to be recovered in a well-conditioned manner [71].

\section{Conclusion}

POFBGs are of increasing interest due to the different material properties of their constituent fibre compared to silica. There remain however a number of challenges that are still to be addressed:

- Understanding the mechanisms for photosensitivity and optimising these - perhaps by the inclusion of photosensitising dopants

- Optimising sensor performance and creating sensors with reproducible characteristics by controlling all stages of the sensor production process - polymerisation, fibre drawing, annealing and inscription

- The lack of a ready source of single mode fibres and components along with the absence of a polymer equivalent of the fusion splicer

There are an increasing number of research groups around the world currently tackling these problems.

\section{References}

[1] Othonos A and Kalli K 1996 Fiber Bragg gratings: fundamentals and applications in telecommunications and sensing (Boston: Artech House Publishers)

[2] Kashyap R 1999 Fiber Bragg gratings (San Diego, CA, USA.: Academic Press) 
[3] Kersey A D, Davis M A, Patrick H J, LeBlanc M, Koo K P, Askins C G, Putnam M A and Friebele E J 1997 Fiber grating sensors Journal of Lightwave Technology 15 1442-63

[4] Rao Y-J 1997 In-fibre Bragg grating sensors Measurement Science and Technology 8 355-75

[5] Bennion I, Williams J A R, Zhang L, Sugden K and Doran N J 1996 UV Written In-Fibre Bragg Gratings Optical and Quantum Electronics 28 93-135

[6] Vasil'ev S A, Medvedkov O I, Korolev I G, Bozhkov A S, Kurkov A S and Dianov E M 2005 Fibre gratings and their applications Quantum Electronics 35 1085-103

[7] Li Z C, Tam H Y, Xu L X and Zhang Q J 2005 Fabrication of long-period gratings in poly(methyl methacrylate-co-methyl vinyl ketone-cobenzyl methacrylate)-core polymer optical fiber by use of a mercury lamp Optics Letters 30 1117-9

[8] Sáez-Rodríguez D, Cruz J L, Johnson I, Webb D J, Large M C J and Argyros A 2010 Water diffusion into UV inscripted Long Period Grating in microstructured polymer fibre leee Sens J $101169-73$

[9] Zubia J and Arrue J 2001 Plastic optical fibres: an introduction to their technological processes and applications Optical Fiber Technology 7 101-40

[10] Ziemann O, Krauser J, Zamzow P E and Daum W 2008 POF handbook: optical short range transmission systems: Springer Science \& Business Media)

[11] Peters K 2011 Polymer optical fiber sensors-a review Smart Materials and Structures 20

[12] Kurkjian C, Krause J and Matthewson M 1989 Strength and Fatigue of Silica Optical Fibers Journal of Lightwave Technology 7 1360-70

[13] Nellen P, Maurona P, Franka A, Sennhausera U, Bohnert K, Pequignotb P, Bodorb P and Brändleb H 2003 Reliability of fiber Bragg grating based sensors for downhole applications Sensors and Actuators A: Physical 103 364-76

[14] Lindner E, Moerbitz J, Chojetzki C, Becker M, Brueckner S, Schuster K, Rothhardt M and Bartelt H 2011 Draw Tower Fiber Bragg Gratings and their use in sensing technology. In: Conference on the Fiber Optic Sensors and Applications VIII, (Orlando, FL

[15] Aressy M 2006 Manufacturing optimisation and mechanical properties of polymer optical fibre. (Birmingham: Birmingham University)

[16] Jiang C, Kuzyk M, Ding J-L, Johns W and Welker D 2002 Fabrication and mechanical behavior of dye-doped polymer optical fiber Journal of Applied Physics 92 04-Dec

[17] Yang D, Yu J, Tao X and Tam H 2004 Structural and mechanical properties of polymeric optical fiber Materials Science and Engineering A364 256-9

[18] Kaye G W and Laby T H 1995 Tables of physical and chemical constants: Longmann)

[19] Brandrup J 1999 Polymer Handbook vol 1\&2: Wiley)

[20] Ye C C, Dulieu-Barton J M, Webb D J, Zhang C, Peng G D, Chambers A R, Lennard F J and Eastop D D 2009 Applications of polymer optical fibre grating sensors to condition monitoring of textiles. In: 20th International Conference on Optical Fibre Sensors, ed J D C Jones (Edinburgh: SPIE) p 75030M

[21] Harbach N G 2008 Fiber bragg gratings in polymer optical fibers. (Lausanne: EPFL)

[22] Zhang W, Webb D and Peng G 2012 Polymer optical fiber Bragg grating acting as an intrinsic biochemical concentration sensor Optics Letters 37 1370-2

[23] Zhang C, Chen X, Webb D J and Peng G-D 2009 Water detection in jet fuel using a polymer optical fibre Bragg grating. In: 20th International Conference on Optical Fibre Sensors, ed J D C Jones (Edinburgh: SPIE) p 750380

[24] Johnson I P, Yuan W, Stefani A, Nielsen K, Rasmussen H K, Khan L, Webb D J, Kalli K and Bang O 2011 Optical fibre Bragg grating recorded in TOPAS cyclic olefin copolymer Electronics Letters 47 271-2

[25] Tagaya A, Koike Y, Kinoshita T, Nihei E, Yamamoto T and Sasaki K 1993 Polymer optical fiber amplifier Applied Physics Letters 63 883-4

[26] Kuzyk M, Paek U and Dirk C 1991 Guest-host polymer fibers for nonlinear optics Applied Physics Letters 59902 
[27] Emiliyanov G, Jensen J B, Bang O, Hoiby P E, Pedersen L H, Kjaer E M and Lindvold L 2007 Localized biosensing with Topas microstructured polymer optical fiber: Erratum Optics Letters 321059

[28] Makino K, Kado T, Inoue A and Koike Y 2012 Low loss graded index polymer optical fiber with high stability under damp heat conditions Optics Express 20 12893-8

[29] Abang A, Saez-Rodriguez D, Nielsen K, Bang O and Webb D J 2013 Connectorisation of fibre Bragg grating sensors recorded in microstructured polymer optical fibre. In: Fifth European Workshop on Optical Fibre Sensors, ed L R Jaroszewicz

[30] Koike Y and Asai M 2009 The future of plastic optical fiber NPG Asia Mater 1 22-8

[31] Abang A and Webb D J 2014 Effects of annealing, pre-tension and mounting on the hysteresis of polymer strain sensors Measurement Science and Technology 25015102

[32] Yuan S, Stefani A, Bache M, Jacobsen T, Rose B, Herholdt-Rasmussen N, Nielsen F, Andersen $\mathrm{S}$, Sørensen O, Hansen $\mathrm{K}$ and Bang $\mathrm{O} 2011$ Improved thermal and strain performance of annealed polymer optical fiber Bragg gratings Optics Communications 284 176-82

[33] Szczurowski M K, Martynkien T, Statkiewicz-Barabach G, Urbanczyk W, Khan L and Webb D J 2010 Measurements of stress-optic coefficient in polymer optical fibers Optics Letters 35 2013-5

[34] Johnson I P, Webb D and Kalli K 2012 Hydrostatic pressure sensing using a polymer optcal fibre Bragg grating. In: 3rd Asia Pacific Optical Sensors Conference, (Sydney, Australia: SPIE)

[35] Salem D R 2001 Structure Formation in Polymeric Fibers: Hanser-Gardner Publications)

[36] Markos C, Stefani A, Nielsen K, Rasmussen H K, Yuan W and Bang O 2013 High-T-g TOPAS microstructured polymer optical fiber for fiber Bragg grating strain sensing at 110 degrees Optics Express 21 4758-65

[37] Dobb H, Webb D J, Kalli K, Argyros A, Large M C J and van Eijkelenborg M A 2005 Continuous wave ultraviolet light-induced fiber Bragg gratings in few- and single-mode microstructured polymer optical fibers Optics Letters 30 3296-8

[38] Tomlinson W J, Kaminow I P, Chandross A, Forck R L and Silfvast W T 1970 Photoinduced refractive index increase in poly(methyl methacrylate) and its applications Applied Physics Letters 16 486-9

[39] Bowden M J, Chandross E A and Kaminow I P 1974 Mechanisms of the photoinduced refractive index increase in Polymethyl Methacrylate Applied Optics 13112

[40] Marotz J 1985 Holographic storage in sensitized polymethyl methacrylate blocks Appl. Phys. B 37 181-7

[41] Moran J M and Kaminow I P 1973 Properties of holographic gratings photoinduced in polymethyl methacrylate Applied Optics 12 1964-70

[42] Kopietz M, Lechner M D, Steinmeier D G, Marotz J, Franke H and Kratzig E 1984 LightInduced Refractive-Index Changes in Polymethylmethacrylate (Pmma) Blocks Polym Photochem 5 109-19

[43] Mitsuoka T, Torikai A and Fueki K 1993 Wavelength sensitivity of the photodegradation of poly(methyl methacrylate) Journal of Applied Polymer Science 47 1027-32

[44] Sáez-Rodríguez D, Nielsen K, Bang O and Webb D J 2014 Photosensitivity mechanism of undoped poly(methyl methacrylate) under UV radiation at $325 \mathrm{~nm}$ and its spatial resolution limit Optics Letters 39 3421-4

[45] Tyler D R 2004 Mechanistic aspects of the effects of stress on the rates of photochemical degradation reactions in polymers Polym. Rev. 44

[46] Wochnowski C, Metev S and Sepold G 2000 UV-laser-assisted modification of the optical properties of polymethylmethacrylate App/ Surf Sci 706-11

[47] Liu H Y, Liu H B, Peng G D and Chu P L 2004 Observation of type I and type II gratings behaviour in polymer optical fiber Optics Communications 16 159-61 
[48] Liu H B, Liu H Y, Peng G D and Chu P L 2004 Novel growth behaviours of fiber Bragg gratings in polymer optical fiber under UV irradiation with low power IEEE Photonics Technology Letters 16 159-61

[49] Bundalo I-L, Nielsen K, Markos C and Bang O 2014 Bragg grating writing in PMMA microstructured polymer optical fibers in less than 7 minutes Optics express 22 5270-6

[50] Laming F P 1971 Holographic grating formation in photopolymers - polymethylmethacrylate Polym. Eng. Sci. 11 421-5

[51] Peng G D, Xiong Z and Chu P L 1999 Photosensitivity and gratings in dye-doped polymer optical fibers Optical Fiber Technology 5 242-51

[52] Yu J, Tao X and Tam H 2004 Trans-4-stilbenemethanol-doped photosensitive polymer fibers and gratings Optics Letters 29 156-8

[53] Kowal D, Statkiewicz-Barabach G, Mergo P and Urbanczyk W 2014 Microstructured polymer optical fiber for long period gratings fabrication using an ultraviolet laser beam Optics Letters $392242-5$

[54] Luo Y, Zhang, Q, Liu H and Peng GD 2010 Gratings fabrication in benzildimethylketal doped photosensitive polymer optical fibers using $355 \mathrm{~nm}$ nanosecond pulsed laser Optics Letters 35

[55] Franke H 1984 Optical recording of refractive-index patterns in doped poly-(methyl methacrylate) films Applied Optics 23 2729-33

[56] Saez-Rodriguez D, Nielsen K, Rasmussen H K, Bang O and Webb D J 2013 Highly photosensitive polymethyl methacrylate microstructured polymer optical fiber with doped core Optics Letters 38 3769-72

[57] TOPAS cyclic olefin copolymer, http::/http://www.topas.com.

[58] Yuan W, Khan L, Webb D J, Kalli K, Rasmussen H K, Stefani A and Bang O 2011 Humidity insensitive TOPAS polymer fiber Bragg grating sensor Optics Express 19 19731-9

[59] Koerdt M, Kibben S, Hesselbach J, Brauner C, Herrmann A S, Vollertsen F and Kroll L 2014 Fabrication and characterization of Bragg gratings in a graded-index perfluorinated polymer optical fiber In: 2nd International Conference on System-Integrated Intelligence: Challenges for Product and Production Engineering Elsevier)

[60] Baum A, Scully P J, Perrie W, Liu D and Lucarini V 2010 Mechanisms of femtosecond laserinduced refractive index modification of poly(methyl methacrylate) J. Opt. Soc. Am. B 27 107-11

[61] Kersey A D and Marrone M J 1994 Fiber Bragg grating high-magnetic-field probe. pp 53-6

[62] Chen X, Zhang C, Webb D J, Peng G-D and Kalli K 2010 Bragg grating in a polymer optical fibre for strain, bend and temperature sensing Measurement Science \& Technology 21 094005

[63] Peng G D and Chu P L 2000 Polymer optical fiber photosensitivities and highly tunable fiber gratings Fiber and Integrated Optics 19 277-93

[64] Brady G, Kalli K, Webb D J, D.A. Jackson L R and Archambault J L 1997 Simultaneous measurement of strain and temperature using the first- and second-order diffraction wavelengths of Bragg gratings IEE Proceedings - Optoelectronics 144 156-61

[65] Rajan G, Liu B, Luo Y, Ambikairajah E and Peng G-D 2013 High Sensitivity Force and Pressure Measurements Using Etched Singlemode Polymer Fiber Bragg Gratings leee Sens J 13 1794800

[66] Rajan G, Noor M Y M, Lovell N H, Ambikaizrajah E, Farrell G and Peng G D 2013 Polymer micro-fiber Bragg grating Optics Letters 38 3359-62

[67] Hu X, Pun C-F J, Tam H-Y, Megret P and Caucheteur C 2014 Highly reflective Bragg gratings in slightly etched step-index polymer optical fiber Optics Express 22 18807-17

[68] Rajan G, Noor M Y M, Ambikairajah E and Peng G-D 2014 Inscription of Multiple Bragg Gratings in a Single-Mode Polymer Optical Fiber Using a Single Phase Mask and Its Analysis leee Sens J 14 2384-8 
[69] Liu H Y, Peng G D and Chu P L 2001 Thermal tuning of polymer optical fiber Bragg gratings IEEE Photonics Technology Letters 13 824-6

[70] Zhang Z F and Tao X M 2012 Synergetic Effects of Humidity and Temperature on PMMA Based Fiber Bragg Gratings Journal of Lightwave Technology 30 841-5

[71] Zhang C, Zhang W, Webb D J and Peng G D 2010 Optical fibre temperature and humidity sensor Electronics Letters 46 643-U63

[72] Carroll K E, Zhang C, Webb D J, Kalli K, Argyros A and Large M C J 2007 Thermal response of Bragg gratings in PMMA microstructured optical fibers Optics Express 15 8844-50

[73] Johnson I P, Webb D J and Kalli K 2011 Utilisation of thermal annealing to record multiplexed FBG sensors in multimode microstructured polymer optical fibre. In: 21st International Conference on Optical Fiber Sensors (OFS21), ed W J Bock, et al. (Ottawa, Canada: SPIE) pp 7753-327

[74] Zhang W and Webb D J 2014 Humidity responsivity of polymer optical fiber Bragg grating sensors Optics Letters 39 3016-29

[75] Zhang W, Webb D J and Peng G D 2012 Investigation Into Time Response of Polymer Fiber Bragg Grating Based Humidity Sensors Journal of Lightwave Technology 30 1090-6

[76] Rajan G, Noor Y M, Liu B, Ambikairaja E, Webb D J and Peng G-D 2013 A fast response intrinsic humidity sensor based on an etched singlemode polymer fiber Bragg grating Sensor Actuat a-Phys 203 107-11

[77] Barbosa-Cánovas G V, Fontana J, A. J. S, S. J. and Labuza T P 2007 Water Activity in FoodsFundamentals and Applications: Blackwell Publishing)

[78] Zhang W, Webb D J, Carpenter M and Williams C 2014 Measuring Water Activity of Aviation Fuel Using a Polymer Optical Fiber Bragg Grating. In: Proc. of SPIE Vol, pp 91574V-1

[79] Johnson I P, Webb D J, Kalli K, Large M C and Argyros A 2010 Multiplexed FBG sensor recorded in multimode microstructured polymer optical fibre. In: Photonic Crystal Fibres, ed K Kalli and W Urbanczyk (Brussels - Photonics Europe: SPIE) p 10

[80] Abang A and Webb D J 2012 Demountable connection for polymer optical fiber grating sensors Optical Engineering 51

[81] Law S H, Harvey J D, Kruhlak R J, Song M, Wu E, Barton G W, van Eijkelenborg M A and Large M C J 2006 Cleaving of microstructured polymer optical fibres Optics Communications 258 193-202

[82] Stefani A, Nielsen K, Rasmussen H K and Bang O 2012 Cleaving of TOPAS and PMMA microstructured polymer optical fibers: Core-shift and statistical quality optimization Optics Communications 285 1825-33

[83] Liu H Y, Liu H B, Peng G D and Chu P L 2006 Polymer optical fibre Bragg gratings based fibre laser Optics Communications 132-5

[84] Kalli K, Dobb H L, Webb D J, Carroll K, Themistos C, Komodromos M, Peng G D, Fang Q and Boyd I W 2007 Development of an electrically tuneable Bragg grating filter in polymer optical fibre operating at $1.55 \mathrm{mu} \mathrm{m}$ Measurement Science \& Technology 18 3155-64

[85] Zhang W, Abang A, Webb D J and Peng G D 2012 Micro-Structured and Specialty Optical Fibres, ed K Kalli and A Mendez

[86] Chen X, Zhang C, Hoe B V, Webb D J, Kalli K, Steenberge G V and Peng G-D 2010 Photonic skin for pressure and strain sensing. In: Optical Sensors and Detection, ed F Berghmans and $A$ G Mignani (Brussels - Photonics Europe: SPIE) p 3

[87] Rajan G, Ramakrishnan M, Semenova Y, Ambikairajah E, Farrell G and Peng G-D 2014 Experimental Study and Analysis of a Polymer Fiber Bragg Grating Embedded in a Composite Material Journal of Lightwave Technology 32 1726-33

[88] Stefani A, Andresen S, Yuan W and Bang O 2012 Dynamic Characterization of Polymer Optical Fibers leee Sens $J 12$ 3047-53 
[89] Stefani A, Andresen S, Yuan W, Herholdt-Rasmussen N and Bang O 2012 High Sensitivity Polymer Optical Fiber-Bragg-Grating-Based Accelerometer leee Photonics Technology Letters 24 763-5

[90] Gallego D and Lamela H 2009 High-sensitivity ultrasound interferometric single-mode polymer optical fiber sensors for biomedical applications Opt. Lett. 34 1807-9

[91] Marques C A F, Bilro L, Kahn L, Oliveira R A, Webb D J and Nogueira R N 2013 Acousto-Optic Effect in Microstructured Polymer Fiber Bragg Gratings: Simulation and Experimental Overview Journal of Lightwave Technology 31 1551-8

[92] Hemighaus G, Boval T, Bacha J, Barnes F, Franklin M, Gibbs L, Hogue N, Jones J, Lesnini D, Lind J and Morris J 2006 Aviation Fuels Technical Review. 